\title{
Low voltage varistor ceramics based on $\mathrm{SnO}_{2}$
}

\author{
S R DHAGE*, V RAVI ${ }^{\dagger}$ and $O$ B YANG \\ School of Environmental and Chemical Engineering, Chonbuk National University, Jeonju 561-756, South Korea \\ ${ }^{\dagger}$ Physical and Materials Chemistry Division, National Chemical Laboratory, Pune 411 008, India
}

MS received 21 May 2007; revised 18 August 2007

\begin{abstract}
The nonlinear current $(I)$-voltage $(V)$ characteristics of tin dioxide doped with either $\mathrm{Nb}_{2} \mathrm{O}_{5}$ and $\mathrm{CoO}$ or $\mathrm{Sb}_{2} \mathrm{O}_{3}$ and $\mathrm{CoO}$ show promising values of nonlinear coefficient $(\alpha)$ values $(\sim 11)$ with low breakdown voltages $\left(E_{\mathrm{B}}, \sim 40 \mathrm{~V} \mathrm{~mm}^{-1}\right)$. The pentavalent antimony or niobium acts as donor and increases the electronic conductivity. The crucial parameter for obtaining low breakdown voltage is the grain size, which depends upon sintering duration and temperature of these oxide ceramics.
\end{abstract}

Keywords. Tin oxide; electronic materials; chemical synthesis; microstructure; X-ray diffraction.

\section{Introduction}

Tin dioxide is an $n$-type semiconductor with a tetragonal crystalline structure similar to the rutile structure. It is difficult to sinter $\mathrm{SnO}_{2}$ without additives to high densities and this property is exploited in gas and humidity sensor applications (Pianaro et al 1995). However, high densification is obtained for tin dioxide with addition of small quantities of transition metal ions (Pianaro et al 1995, 1997; Cerri et al 1996). It has already been reported (Pianaro et al 1997; Ravi and Date 2001) that polycrystalline dense ceramics based on $\mathrm{SnO}_{2}-\mathrm{CoO}-\mathrm{Nb}_{2} \mathrm{O}_{5}-\mathrm{Cr}_{2} \mathrm{O}_{3}$ system shows highly nonlinear $I-V$ characteristics. The three important parameters that characterize a varistor are the nonlinear coefficient $(\alpha)$, breakdown voltage $\left(E_{\mathrm{B}}\right)$ and energy absorption capability. The reported $\alpha$ for doped tin dioxide is $>20$ with $E_{\mathrm{B}} \sim 8000 \mathrm{~V} \mathrm{~cm}^{-1}$. Recently, Bueno et al (2001) compared the grain boundary barrier properties of tin dioxide with that of varistors based on $\mathrm{ZnO}$ ceramics. Though $\mathrm{ZnO}$ shows excellent varistor property, the drawback is its high breakdown voltage (Gupta 1990; Clarke 1999). Low voltage varistors based on $\mathrm{SrTiO}_{3}$ (Fujimoto et al 1985) and $\mathrm{SnO}_{2}-\mathrm{TiO}_{2}$ systems (Yan and Rhodes 1982; Bueno et al 2002; Dhage et al 2003) have already been reported in the literature. In the case of $\mathrm{SnO}_{2}$ $\mathrm{TiO}_{2}$ solid solution, the samples were heated at $1723 \mathrm{~K}$ to obtain large grain growth $(>15 \mu \mathrm{m})$ and $\alpha$ values are in the range 7-9. However, until now, there is no report on low voltage varistor based on $\mathrm{SnO}_{2}$ system. Here we report the varistor characteristics of doped tin dioxide with low breakdown voltage.

*Author for correspondence (sanjay.dhage@gmail.com)

\section{Experimental}

$\mathrm{SnO}_{2}$ containing additives were prepared by the wet chemical method. The compositional formulas prepared are given in table 1. $\mathrm{SnCl}_{4}$ was diluted using ice-cold distilled water and mixed with required quantities of $\mathrm{Co}\left(\mathrm{NO}_{3}\right)_{2} \cdot 6 \mathrm{H}_{2} \mathrm{O}$ and $\mathrm{Sb}_{2} \mathrm{O}_{3}$ dissolved in $\mathrm{HCl}$ or $\mathrm{Nb}_{2} \mathrm{O}_{5}$. The standard ammonia solution was then added dropwise to the above solution with constant stirring until $\mathrm{pH}$ was $\sim 10$. The precipitate was filtered, washed free of anions and dried in the oven. The oven dried powders were calcined at $873 \mathrm{~K}$ for $12 \mathrm{~h}$. The calcined powders were mixed with a binder (poly vinyl alcohol, $2 \mathrm{wt} \%$ ) and pelletized (15 $\mathrm{mm}$ dia, $1 \mathrm{~mm}$ thick) at a uniform pressure of 2-3 metric tons. The pellets were sintered at $1573 \mathrm{~K}$ for $15 \mathrm{~h}$ to allow reasonable grain growth. The sintered pellets were polished and ohmic silver contacts were obtained by curing Ag-paste and annealed at $873 \mathrm{~K}$ for $30 \mathrm{~min}$. The structure related phase determination was studied by Philip 1730 X-ray diffractometer. The microstructure of the sintered pellets was observed using a Leica Cambridge 440 microscope. The $I-V$ characteristics were measured by using Keithley electrometer, 6517A. It also contained inbuilt $1 \mathrm{kV}$ d.c. power supply so that there was no need of external power supply in the circuit. The current-voltage relation of a varistor is given by the equation

$$
J=(E / C)^{\alpha},
$$

where $J$ is the current density, $E$ the applied field, $C$ the proportionality constant and $\alpha$ the nonlinear coefficient. The $I-V$ curves were plotted on $\log -\log$ scale from which the slope of the curve gave the value of $\alpha$. The important parameter, $E_{\mathrm{B}}$ (breakdown voltage), was taken as the field applied when current flowing through the varistor was $1 \mathrm{~mA}$. Since Schottky type grain boundary barriers 
Table 1. Characteristic parameters of doped tin dioxide ceramics.

\begin{tabular}{lcrcccc}
\hline $\begin{array}{l}\text { Sample } \\
\text { identity }\end{array}$ & Composition & $\alpha$ & $E_{\mathrm{B}}\left(\mathrm{V} \mathrm{mm}^{-1}\right)$ & $\Phi_{\mathrm{B}}(\mathrm{eV})$ & $\begin{array}{c}\text { Relative } \\
\text { density }(\%)\end{array}$ & $\begin{array}{c}\text { Average grain } \\
\text { size }(\mu \mathrm{m})\end{array}$ \\
\hline $\mathrm{SCA}-1$ & $(98 \cdot 99) \% \mathrm{SnO}_{2}+1 \% \mathrm{CoO}+0 \cdot 01 \% \mathrm{Sb}_{2} \mathrm{O}_{3} / 2$ & 10 & 41 & $0 \cdot 30$ & $96 \cdot 2$ & 10 \\
$\mathrm{SCA}-2$ & $(98.95) \% \mathrm{SnO}_{2}+1 \% \mathrm{CoO}+0 \cdot 05 \% \mathrm{Sb}_{2} \mathrm{O}_{3} / 2$ & 6 & 26 & $0 \cdot 29$ & $94 \cdot 8$ & 12 \\
$\mathrm{SCA}-3$ & $(98 \cdot 9) \% \mathrm{SnO}_{2}+1 \% \mathrm{CoO}+0 \cdot 1 \% \mathrm{Sb}_{2} \mathrm{O}_{3} / 2$ & 8 & 41 & $0 \cdot 34$ & $95 \cdot 9$ & 11 \\
$\mathrm{SCN}-3$ & $(98 \cdot 9) \% \mathrm{SnO}_{2}+1 \% \mathrm{CoO}+0 \cdot 1 \% \mathrm{Nb}_{2} \mathrm{O}_{5} / 2$ & 7 & 23 & $0 \cdot 31$ & $95 \cdot 6$ & 11 \\
\hline
\end{tabular}

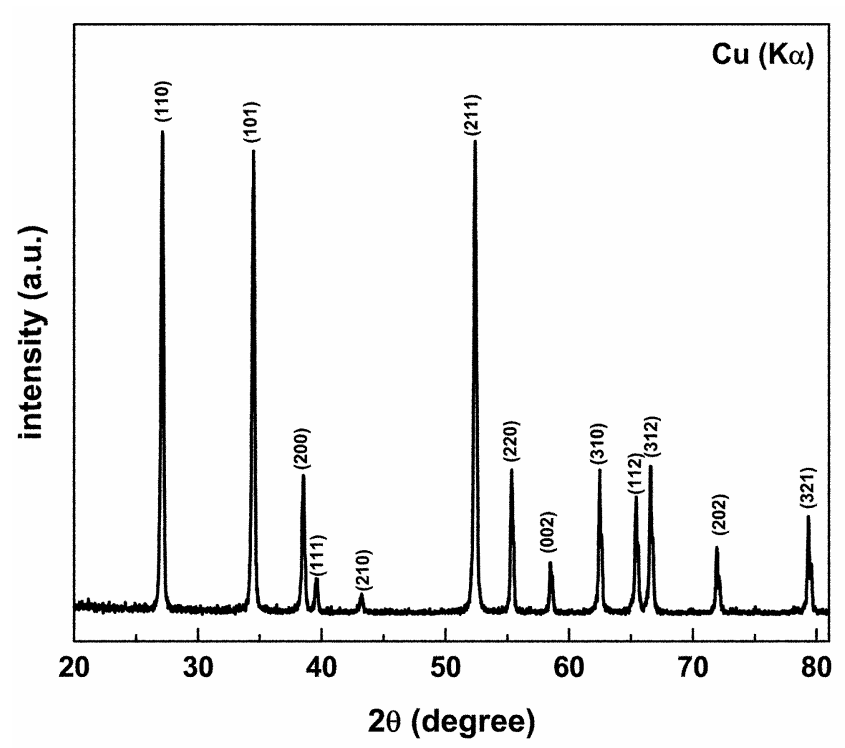

Figure 1. XRD of doped tin dioxide.

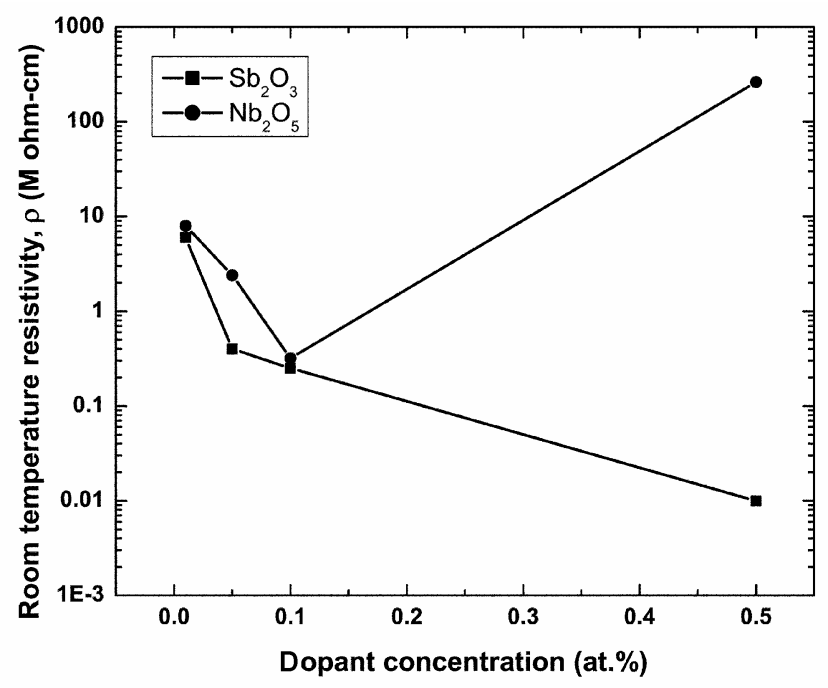

Figure 2. Variation of room temperature resistivity with donor content for $\mathrm{CoO}$ doped tin dioxide.

were present in the present samples, the current density in ohmic region of varistor was related to the electric field and the temperature given by the equation (Pianaro et al 1997)

$$
J=A T^{2} \exp \left[\left(\beta E^{1 / 2}-\Phi_{\mathrm{B}}\right) / k T\right],
$$

where $A=4 \rho e m k^{2} / h^{3}$, is Richardson's constant, $e$ the electron charge, $m$ the electron mass, $k$ the Boltzmann constant, $h$ the plank constant, $\Phi_{\mathrm{B}}$ the interface barrier height, and $\beta$ a constant related to the relationship given in the equation

$$
\beta \alpha 1 /(r \times \omega)
$$

where $r$ is the grain number per unit length and $\omega$ the barrier width, making the current density in ohmic region and keeping the temperature of the tested varistor constant. For two different applied fields, the following equations are used.

$$
\begin{aligned}
& J_{1}=A T^{2} \exp \left[\left(\beta E_{1}^{1 / 2}-\Phi_{\mathrm{B}}\right) / k T\right], \\
& J_{2}=A T^{2} \exp \left[\left(\beta E_{2}^{1 / 2}-\Phi_{\mathrm{B}}\right) / k T\right],
\end{aligned}
$$

The values of $\Phi_{\mathrm{B}}$ and $\beta$ can be calculated from the above equations.

\section{Results and discussion}

Figure 1 shows the X-ray diffractogram (XRD) recorded for the present sample. No second phases are found and all the lines are corresponding to $\mathrm{SnO}_{2}$ tetragonal rutile type phase. It is also to be noted that the concentrations of dopants added are too small to be detected by X-rays. The variation of room temperature resistivity with donor content is depicted in figure 2 . The resistivity decreases continuously for antimony doped sample whereas it increases after a minimum value for niobium doped specimens. For identical conditions of processing, low voltage varistor action is observed over a range of antimony doping whereas low breakdown voltage is found at a specific concentration $(0 \cdot 1 \mathrm{at} \%)$ of niobium only. This is evident from the resistivity-donor content relations for these ceramics. The obtained values of barrier height, breakdown voltage, nonlinear coefficient, relative sinter density and grain size are given in table 1 . The $I-V$ curves for the samples containing $\mathrm{Sb}$ and $\mathrm{Nb}$ are illustrated in figure 3 . The calculated $\alpha$ value for $\mathrm{Sb}$ and $\mathrm{Co}$ doped $\mathrm{SnO}_{2}$ is 11 and for $\mathrm{Nb}$ and $\mathrm{Co}$ doped $\mathrm{SnO}_{2}$ is 7 and their breakdown voltages are in the range of $40-20 \mathrm{~V} \mathrm{~mm}^{-1}$ as shown in table 1. The microstructure of $\mathrm{Sb}$ and $\mathrm{Nb}$ doped samples are shown in figures 4 (a) and (b), the grain size is $\sim 12 \mu \mathrm{m}$ 
and $\sim 11 \mu \mathrm{m}$, respectively. No second phases were found at grain boundaries supporting XRD results.

The varistor action observed in polycrystalline $\mathrm{ZnO}$ ceramics is explained by the presence of Schottky type

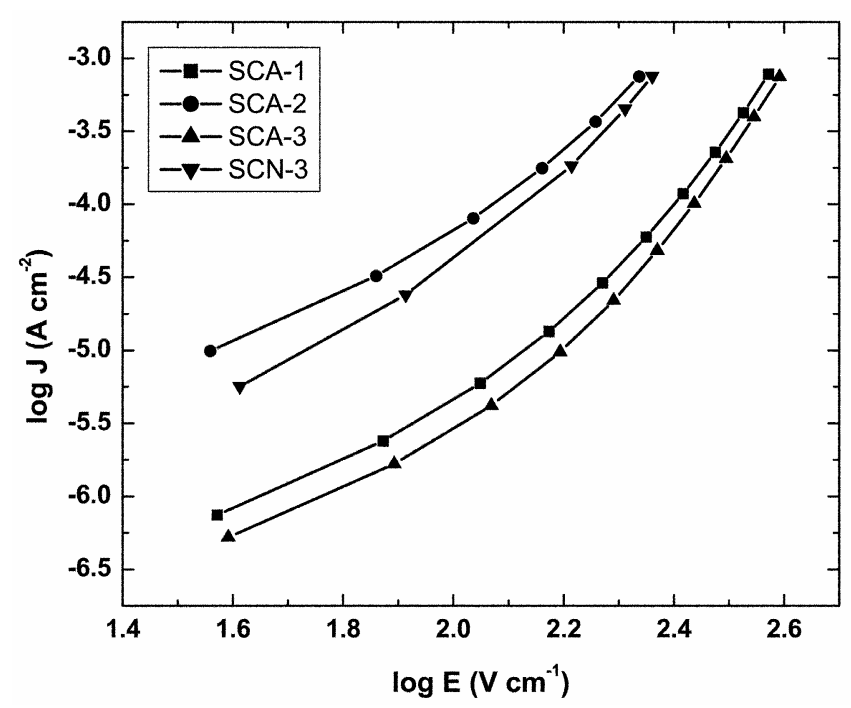

Figure 3. $I-V$ characteristics of doped tin dioxide ceramics.
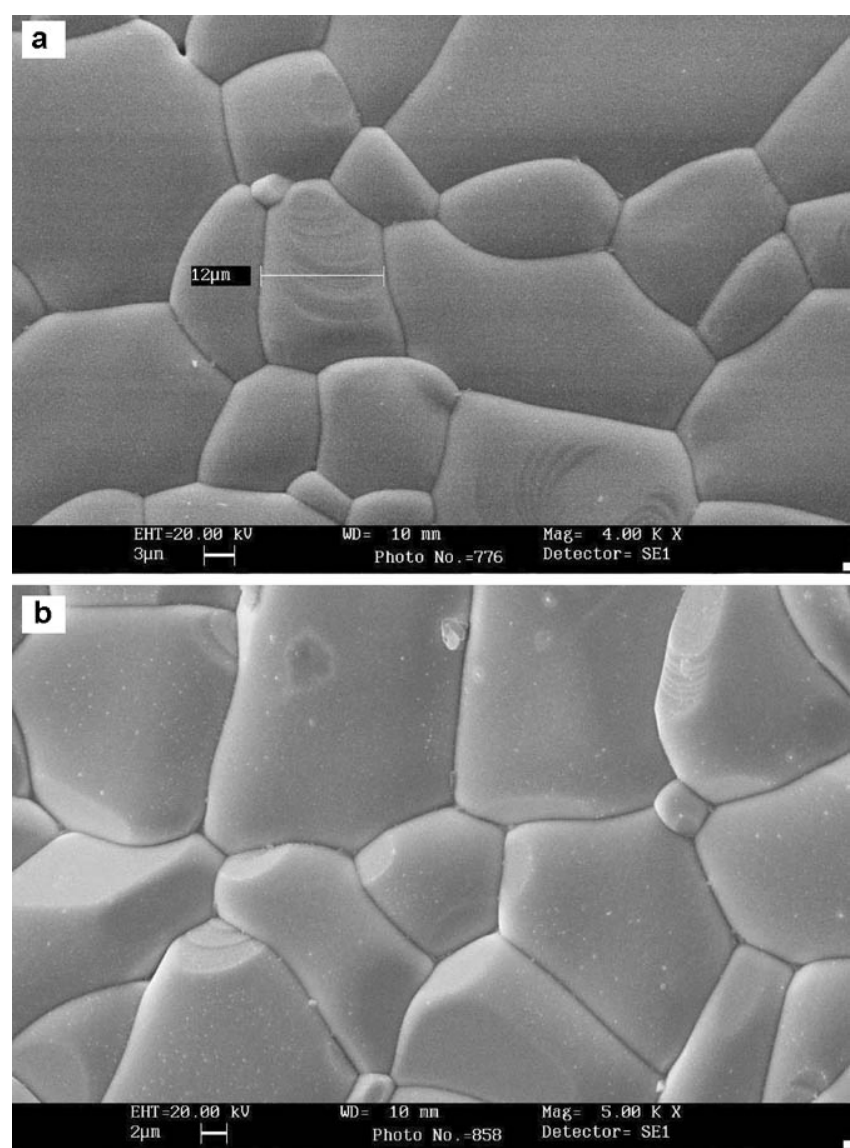

Figure 4. Microstructure of doped tin dioxide ceramics: (a) SCA-2 and (b) SCN-3. energy barrier at the grain boundaries. In the present case the acceptor like surface states formed by $\mathrm{CoO}$ will lead to formation of energy barrier at the grain boundaries. This is unlike other tin oxide system reported by us (Dhage et al 2002) wherein the presence of rare earth ion at the grain boundaries leads to formation of grain boundary defect states. There the ionic radius of $\mathrm{La}^{3+}$ ion being larger than $\mathrm{Sn}^{4+}$ ion, it prefers grain boundary site. This modification resulted in high $\alpha$ values. As mentioned in the introduction the addition of small quantities of transition metal oxides such as $\mathrm{CoO}$ to tin oxide helps in its densification. For example, with $1 \mathrm{~mol} \% \mathrm{CoO}$, the sinter density is $>94 \%$ when sintered at $1573 \mathrm{~K}$ for $1 \mathrm{~h}$. The $\mathrm{Co}^{2+}$ ion having lower valence creates oxygen vacancies in tin oxide lattice, which is rate-determining step for sintering. The reaction may be written as

$$
\mathrm{CoO} \stackrel{\mathrm{SnO}_{2}}{\longrightarrow} \mathrm{Co}_{\mathrm{Sn}}+\mathrm{V}_{\mathrm{o}}^{*}+\mathrm{O}_{\mathrm{o}}^{\mathrm{x}}
$$

The ionic radius of cobaltous ion being similar to $\mathrm{Sn}^{4+}$ ion, it may substitutionally replace latter in tin oxide lattice. Co segregation at grain boundary is reported with the help of EDX, TEM and impedance analysis (Wang et al 2000; Bacelar et al 2002; Cassia et al 2005). The incorporation of either antimony oxide or niobium oxide increases electronic conductivity of tin oxide. At lower concentrations, antimony is present as pentavalent and acts as a donor. The defect reaction may be given as

$$
\mathrm{Sb}_{2} \mathrm{O}_{5} \stackrel{\mathrm{SnO}_{2}}{\longrightarrow} 2 \mathrm{Sb}_{\mathrm{Sn}}+2 e^{\prime}+4 \mathrm{O}_{\mathrm{o}}^{\mathrm{x}}+1 / 2 \mathrm{O}_{2} \text {. }
$$

The present varistor system contains less number of additives when compared to $\mathrm{ZnO}$ ceramics wherein a variety of metal oxides are added in small quantities to achieve optimum properties.

\section{Conclusions}

Varistor with low breakdown voltages $\left(E_{\mathrm{B}}, \sim 40 \mathrm{~V} \mathrm{~mm}^{-1}\right)$ are obtained on doped tin dioxide system. The same ceramics show higher breakdown voltages when the grain size is decreased.

\section{Acknowledgement}

Brain Korea 21 project through the Centre for Future Energy Materials and Devices and Chonbuk National University are acknowledged for financial support.

\section{References}

Bacelar W K, Oliveira M M, Souza V C, Longo E, Leite E R and Varela J A 2002 J. Mater. Sci.: Mater. Electron. 13409

Bueno P R, Cassia-Santors M R, Simoes L G, Gomes J W and Longo E 2002 J. Am. Ceram. Soc. 85282

Bueno P R, Leite E R, Oliveria M M, Orlandi M O and Longo E 2001 Appl. Phys. Lett. 7948 
Cassia M R et al 2005 Mater. Chem. Phys. 901

Cerri J A, Leite E R, Gouvea D, Longo E and Varela J A 1996 J. Am. Ceram. Soc. 79799

Clarke D R 1999 J. Am. Ceram. Soc. 82485

Dhage S R, Ravi V and Date S K 2002 Mater. Lett. 56727

Dhage S R, Samuel V and Ravi V 2003 J. Electroceram. 11 81

Fujimoto M, Chiang Y M, Roshko A and Kingery W D $1985 \mathrm{~J}$. Am. Ceram. Soc. 68 C300
Gupta T K 1990 J. Am. Ceram. Soc. 731817

Pianaro S A, Bueno P R, Longo E and Varela J A 1995 J. Mater. Sci. Lett. 14692

Pianaro S A, Bueno P R, Olivi P, Longo E and Varela J A 1997 J. Mater. Sci. Lett. 16634

Ravi V and Date S K 2001 Bull. Mater. Sci. 24483

Wang Y J, Wang J F, Li C P, Chen H C, Su W B, Zhong W L, Zhang P L and Zhao L Y 2000 Eur. Phys. J. AP 11155

Yan M F and Rhodes W W 1982 Appl. Phys. Lett. 40536 